\title{
PRINCIPAL CONGRUENCES OF PSEUDOCOMPLEMENTED DISTRIBUTIVE LATTICES
}

\author{
HARRY LAKSER ${ }^{1}$ \\ ABSTRACT. Principal congruences of pseudocomplemented dis- \\ tributive lattices are characterized. This characterization is used to \\ give a new proof of the congruence extension property ana to show \\ that the meet of two principal congruences is always principal in an \\ equational class of pseudocomplemented distributive lattices if and \\ only if the class is a subclass of the class of Stone algebras.
}

1. Introduction. In the series of papers [3], [4], and [6], the structure of pseudocomplemented distributive lattices was rather thoroughly discussed. One topic untouched, though, was the characterization of (pseudocomplementation-preserving) principal congruence relations in pseudocomplemented distributive lattices in a form analogous to the wellknown characterization of principal congruence relations in distributive lattices (see [2, §9]). In Theorem 1 such a characterization is given, and it yields, via a result of A. Day [1], a new proof of the congruence extension property (see [3]).

It has long been known that in any distributive lattice the meet of two principal congruence relations is principal (this is implicit in [5]). Kirby Baker told me in a conversation that Stone algebras have the same property and asked whether the class of all pseudocomplemented distributive lattices has this property. This question is answered in Theorem 2.

I first obtained the characterization in Theorem 1 from the free pseudocomplemented distributive lattice generated by a comparable pair of elements. I should like to thank Barry Wolk for applying his impressive skill in computer programming to find that pseudocomplemented distributive lattice.

2. The results. The notations in this paper are those of [3], [4] and [6]. Recall that if $L$ is a pseudocomplemented distributive lattice then the subset $S(L)=\left\{x^{*} \mid x \in L\right\}$, the skeleton of $L$, is a Boolean algebra whose meet operation is $\wedge$, the meet in $L$, whose complementation is *, and whose join

Presented to the Society, February 7, 1972; received by the editors March 23, 1972. AMS (MOS) subject classifications (1970). Primary 06A35; Secondary 08A25.

Key words and phrases. Principal congruence, congruence extension property.

${ }^{1}$ This research was supported by the National Research Council of Canada.

(c) American Mathematical Society 1973 
operation $\cup$ is defined by $a \cup b=\left(a^{*} \wedge b^{*}\right)^{*}$. Clearly, in any Boolean algebra an equivalence relation preserving meet and complementation is a congruence relation; this obvious fact will be used subsequently several times.

For any pair $a, b$ in a pseudocomplemented distributive lattice, $\Theta(a, b)$ denotes the principal congruence of pseudocomplemented distributive lattices (*-congruence) generated by $a, b$. Clearly $\Theta(a, b)=\Theta(a \wedge b, a \vee b)$; thus we need only characterize $\Theta(a, b)$ for comparable $a, b$. We denote by $\Theta_{\text {Lat }}(a, b)$ the principal congruence of distributive lattices generated by $a$, $b$; that is, $\Theta_{\text {Lat }}(a, b)$ has the substitution property for $\wedge$ and $\vee$, but not necessarily for $*$. Recall that if $a \leqq b$ then $x \equiv y\left(\Theta_{\text {Lat }}(a, b)\right)$ if and only if $x \wedge a=y \wedge a$ and $x \vee b=y \vee b$.

LEMMA 1. Let $L$ be a pseudocomplemented distributive lattice, let $a, b \in L$, and let $a \leqq b$. Then

$$
\Theta(a, b)=\Theta_{\mathrm{Lat}}(a, b) \vee \Theta_{\mathrm{Lat}}\left(\left(a^{*} \wedge b\right)^{*}, 1\right) .
$$

ProOF. If $\Phi$ is a *-congruence of $L$ and $a \equiv b(\Phi)$ then $a^{*} \wedge b \equiv 0(\Phi)$ and so $\left(a^{*} \wedge b\right)^{*} \equiv 1(\Phi)$; thus

$$
\Theta_{\text {Lat }}(a, b) \vee \Theta_{\text {Lat }}\left(\left(a^{*} \wedge b\right)^{*}, 1\right) \leqq \Theta(a, b) .
$$

To conclude the proof we need only show that $\Theta_{\mathrm{Lat}}(a, b) \vee \Theta_{\mathrm{Lat}}\left(\left(a^{*} \wedge b\right)^{*}, 1\right)$ has the substitution property with respect to the operation *. Let $x \equiv y$ $\left(\Theta_{\text {Lat }}(a, b) \vee \Theta_{\text {Lat }}\left(\left(a^{*} \wedge b\right)^{*}, 1\right)\right)$. Then there is a sequence $x=x_{0}, x_{1}, \cdots$, $x_{n}=y$ of elements of $L$ such that, for each $i=0, \cdots, n-1$, either $x_{i} \equiv$ $x_{i+1}\left(\Theta_{\text {Lat }}(a, b)\right)$ or $x_{i} \equiv x_{i+1}\left(\Theta_{\text {Lat }}\left(\left(a^{*} \wedge b\right)^{*}, 1\right)\right)$.

Now $x_{i} \equiv x_{i+1}\left(\Theta_{\text {Lat }}\left(\left(a^{*} \wedge b\right)^{*}, 1\right)\right)$ implies $x_{i} \wedge\left(a^{*} \wedge b\right)^{*}=x_{i+1} \wedge\left(a^{*} \wedge b\right)^{*}$ and thus, using the identity

$$
(u \wedge v)^{* *}=u^{* *} \wedge v^{* *}, \quad x_{i}^{* *} \wedge\left(a^{*} \wedge b\right)^{*}=x_{i+1}^{* *} \wedge\left(a^{*} \wedge b\right)^{*} .
$$

Now $x_{i}^{* *}, x_{i+1}^{* *},\left(a^{*} \wedge b\right)^{*} \in S(L)$ and, for each $u \in L, u^{*}$ is the complement of $u^{* *}$ in the Boolean algebra $S(L)$. We conclude that $x_{i}^{*} \wedge\left(a^{*} \wedge b\right)^{*}=$ $x_{i+1}^{*} \wedge\left(a^{*} \wedge b\right)^{*}$. We have thus shown that $x_{i} \equiv x_{i+1}\left(\Theta_{\text {Lat }}\left(\left(a^{*} \wedge b\right)^{*}, 1\right)\right)$ implies $x_{i}^{*} \equiv x_{i+1}^{*}\left(\Theta_{\text {Lat }}\left(\left(a^{*} \wedge b\right)^{*}, 1\right)\right)$.

On the other hand, $x_{i} \equiv x_{i+1}\left(\Theta_{\text {Lat }}(a, b)\right)$ implies that $x_{i} \wedge a=x_{i+1} \wedge a$ and $x_{i} \vee b=x_{i+1} \vee b$, which imply

$$
x_{i}^{* *} \wedge a^{* *}=x_{i+1}^{* *} \wedge a^{* *} \text { and } x_{i}^{*} \wedge b^{*}=x_{i+1}^{*} \wedge b^{*} .
$$

Since $S(L)$ is a Boolean algebra these latter imply that

$$
x_{i}^{*} \wedge\left(a^{*} \wedge b^{* *}\right)^{*}=x_{i+1}^{*} \wedge\left(a^{*} \wedge b^{* *}\right)^{*} .
$$


But $\left(a^{*} \wedge b\right)^{*}=\left(a^{*} \wedge b\right)^{* * *}=\left(a^{* * *} \wedge b^{* *}\right)^{*}=\left(a^{*} \wedge b^{* *}\right)^{*}$; we thus conclude that

$$
x_{i}^{*} \wedge\left(a^{*} \wedge b\right)^{*}=x_{i+1}^{*} \wedge\left(a^{*} \wedge b\right)^{*} .
$$

Thus $x_{i} \equiv x_{i+1}\left(\Theta_{\text {Lat }}(a, b)\right)$ implies $x_{i}^{*} \equiv x_{i+1}^{*}\left(\Theta_{\text {Lat }}\left(\left(a^{*} \wedge b\right)^{*}, 1\right)\right)$.

We thus conclude that $x_{i}^{*} \equiv x_{i+1}^{*}\left(\Theta_{\text {Lat }}\left(\left(a^{*} \wedge b\right)^{*}, 1\right)\right)$ for all $i=0, \cdots$, $n-1$. Thus $x \equiv y \quad\left(\Theta_{\mathrm{Lat}}(a, b) \vee \Theta_{\mathrm{Lat}}\left(\left(a^{*} \wedge b\right)^{*}, 1\right)\right)$ implies $x^{*} \equiv y^{*}$ $\left(\Theta_{\text {Lat }}\left(\left(a^{*} \wedge b\right)^{*}, 1\right)\right)$; consequently $\Theta_{\text {Lat }}(a, b) \vee \Theta_{\text {Lat }}\left(\left(a^{*} \wedge b\right)^{*}, 1\right)$ preserves * and so the proof is complete.

We immediately conclude

THEOREM 1. Let $L$ be a pseudocomplemented distributive lattice and let $a, b \in L$ with $a \leqq b$. If $x, y \in L$ then $x \equiv y(\Theta(a, b))$ if and only if $x \wedge a=y \wedge a$ and $(x \vee b) \wedge\left(a^{*} \wedge b\right)^{*}=(y \vee b) \wedge\left(a^{*} \wedge b\right)^{*}$.

Proof. $L / \Theta_{\text {Lat }}\left(\left(a^{*} \wedge b\right)^{*}, 1\right)$ is a distributive lattice. For each $u \in L$, let $[u]$ denote the corresponding congruence class in $L / \Theta_{\mathrm{Lat}}\left(\left(a^{*} \wedge b\right)^{*}, 1\right)$. Then, by Lemma $1, x \equiv y(\Theta(a, b))$ if and only if

$$
[x] \equiv[y]\left(\Theta_{\mathrm{Lat}}([a],[b])\right) \quad \text { in } L / \Theta_{\mathrm{Lat}}\left(\left(a^{*} \wedge b\right)^{*}, 1\right)
$$

if and only if

if and only if

$$
\begin{aligned}
& x \wedge a \equiv y \wedge a\left(\Theta_{\text {Lat }}\left(\left(a^{*} \wedge b\right)^{*}, 1\right)\right) \text { and } \\
& x \vee b \equiv y \vee b\left(\Theta_{\text {Lat }}\left(\left(a^{*} \wedge b\right)^{*}, 1\right)\right)
\end{aligned}
$$

$$
\begin{aligned}
x \wedge a \wedge\left(a^{*} \wedge b\right)^{*} & =y \wedge a \wedge\left(a^{*} \wedge b\right)^{*} \text { and } \\
(x \vee b) & \wedge\left(a^{*} \wedge b\right)^{*}=(y \vee b) \wedge\left(a^{*} \wedge b\right)^{*} .
\end{aligned}
$$

Since $a \leqq\left(a^{*} \wedge b\right)^{*}$, the theorem follows.

COROllary (G. GRÄTZER AND H. LAKSER [3]). The equational class of pseudocomplemented distributive lattices has the congruence extension property.

Proof. In [1], A. Day proved that if an equational class has the property that in each algebra in the class $x \equiv y \Theta(a, b)$ is determined by equations involving only $x, y, a, b$ then the congruence extension property holds in the equational class. In our case, by Theorem 1 , these equations are

and

$$
x \wedge a \wedge b=y \wedge a \wedge b
$$

$(x \vee a \vee b) \wedge\left((a \wedge b)^{*} \wedge(a \vee b)\right)^{*}=(y \vee a \vee b) \wedge\left((a \wedge b)^{*} \wedge(a \vee b)\right)^{*}$

(for general, not necessarily comparable, $a$ and $b$ ), proving the corollary. 
For the case of Stone algebras (pseudocomplemented distributive lattices satisfying the identity $\left.\left(u^{*} \wedge v^{*}\right)^{*}=u^{* *} \vee v^{* *}\right)$, Lemma 1 is particularly simple.

Lemma 2. Let $L$ be $a$ Stone algebra and let $a, b \in L$ with $a \leqq b$. Then $\Theta(a, b)=\Theta_{\text {Lat }}\left(a \vee b^{*}, b \vee a^{*}\right)$.

Proof. Clearly $a \vee b^{*} \equiv b \vee a^{*}(\Theta(a, b))$. By Lemma 1 we need only show that $a \equiv b\left(\Theta_{\mathrm{Lat}}\left(a \vee b^{*}, b \vee a^{*}\right)\right)$ and $\left(a^{*} \wedge b\right)^{*} \equiv 1\left(\Theta_{\mathrm{Lat}}\left(a \vee b^{*}, b \vee a^{*}\right)\right)$ to conclude the proof. Now $b \wedge\left(b \vee a^{*}\right)=b$ and $b \wedge\left(a \vee b^{*}\right)=(b \wedge a) \vee\left(b \wedge b^{*}\right)=a$; thus $b \equiv a\left(\Theta_{\mathrm{Lat}}\left(a \vee b^{*}, b \vee a^{*}\right)\right)$. Also, $\left(a^{*} \wedge b\right)^{*}=\left(a^{*} \wedge b^{* *}\right)^{*}=a^{* *} \vee b^{*} \geqq$ $a \vee b^{*}$ and $a^{* *} \vee b^{*} \vee b \vee a^{*}=1$ since $a^{* *} \vee a^{*}=1$; thus $\left(a^{*} \wedge b\right)^{*} \equiv 1$ $\left(\Theta_{\mathrm{Lat}}\left(a \vee b^{*}, b \vee a^{*}\right)\right)$, completing the proof of the lemma.

Now the meet of two principal congruence relations in a distributive lattice is principal (implicit in [5]). Indeed, if $a \leqq b$ and $c \leqq d$ the formula is $\Theta_{\mathrm{Lat}}(a, b) \wedge \Theta_{\mathrm{Lat}}(c, d)=\Theta_{\mathrm{Lat}}((a \wedge d) \vee(b \wedge c), b \wedge d)$. We thus get the immediate corollary.

COROLlARY (K. BAKER). In any Stone algebra the meet of two principal *-congruences is a principal *-congruence.

We now show that the class of Stone algebras is the largest equational class of pseudocomplemented distributive lattices in which the meet of any two principal *-congrunces is principal.

THEOREM 2. If $\mathscr{K}$ is an equational class of pseudocomplemented distributive lattices then the meet of any two principal *-congruences is principal in all algebras of $\mathscr{K}$ if and only if $\mathscr{K}$ is a subclass of the class of all Stone algebras.

ProOF. By the corollary to Lemma 2 we need only exhibit a pseudocomplemented distributive lattice in $\mathscr{B}_{2}$, the equational class covering that of Stone algebras (see [6]), in which the meet of two principal congruences is not principal. We consider the pseudocomplemented distributive lattice $L$ depicted in Figure 1. It is easy to see that $L \in \mathscr{B}_{2}$. We show that $\Theta(a \wedge c) \wedge \Theta(b, c)$ is not a principal *-congruence. Clearly $a^{* *} \equiv c^{* *}=1$ $(\Theta(a, c))$ and $b^{* *} \equiv c^{* *}=1(\Theta(b, c))$. Thus $a^{* *} \vee b^{* *} \equiv 1(\Theta(a, c) \wedge \Theta(b, c))$. Indeed, the nontrivial congruence classes of $\Theta(a, c) \wedge \Theta(b, c)$ are $\left\{a \vee b, c \wedge\left(b \vee a^{* *}\right), c \wedge\left(a \vee b^{* *}\right), c\right\},\left\{a, c \wedge a^{* *}\right\},\left\{b, c \wedge b^{* *}\right\},\left\{a^{* *} \vee b, a^{* *} \vee c\right\}$, $\left\{b^{* *} \vee a, b^{* *} \vee c\right\}$, and $\left\{a^{* *} \vee b^{* *}, 1\right\}$.

Since $\Theta(a, c) \wedge \Theta(b, c)$ leaves $S(L)=\left\{0, a^{* *}, b^{* *}, 1\right\}$ untouched the relation $x \equiv y(\Theta(a, c) \wedge \Theta(b, c))$ implies that $\left(x^{*} \wedge y\right)^{*}=1$. Thus, if there were $x, y \in L, x \leqq y$, such that $\Theta(a, c) \wedge \Theta(b, c)=\Theta(x, y)$ then we would conclude, by Lemma 1 , that $\Theta(a, c) \wedge \Theta(b, c)=\Theta_{\text {Lat }}(x, y)$. Clearly 


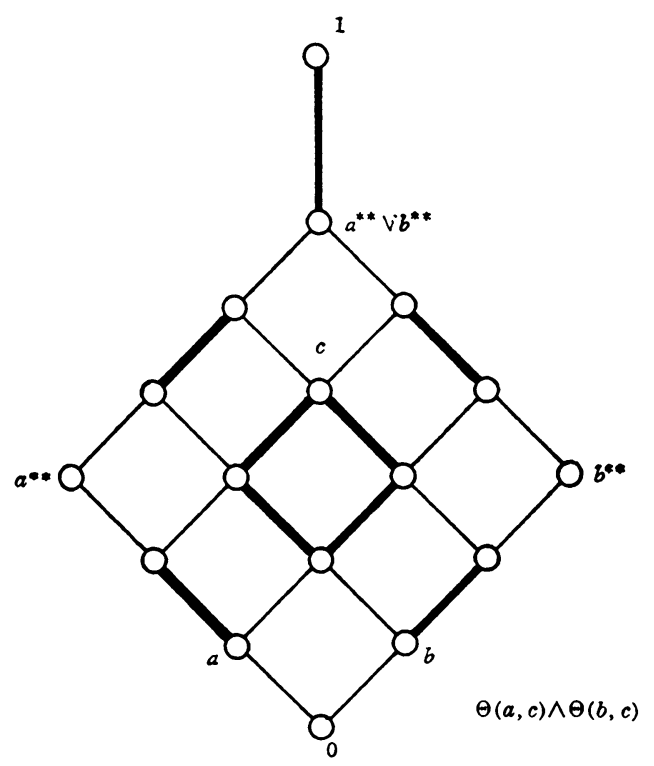

FIGURE 1

$\Theta(a, c) \wedge \Theta(b, c)$ is not a principal congruence of distributive lattices, proving the theorem.

\section{REFERENCES}

1. A. Day, A note on the congruence extension property, Algebra Universalis 1 (1971), 234-235.

2. G. Grätzer, Lattice theory: First concepts and distributive lattices, Freeman, San Francisco, Calif., 1971.

3. G. Grätzer and H. Lakser, The structure of pseudocomplemented distributive lattices. II. Congruence extension and amalgamation, Trans. Amer. Math. Soc. 156 (1971), 343358. MR 43 \#124.

4. — The structure of pseudocomplemented distributive lattices. III. Injectives and absolute subretracts, Trans. Amer. Math. Soc. 169 (1972), 475-487.

5. G. Grätzer and E. T. Schmidt, On the generalized Boolean algebra generated by a distributive lattice, Nederl. Akad. Wetensch. Proc. Ser. A. $61=$ Indag. Math. 20 (1958), 547-553. MR 20 \#6991.

6. H. Lakser, The structure of pseudocomplemented distributive lattices. I. Subdirect decomposition, Trans. Amer. Math. Soc. 156 (1971), 335-342. MR 43 \#123.

Department of Mathematics, University of Manitoba, Winnipeg, Manitoba, CANADA 